\title{
Selective stimulation of VEGFR-1 prevents oxygen-induced retinal vascular degeneration in retinopathy of prematurity
}

\author{
Shu-Ching Shih, Meihua Ju, Nan Liu, and Lois E.H. Smith \\ Department of Ophthalmology, Harvard Medical School and Children's Hospital Boston, Boston, Massachusetts, USA
}

\begin{abstract}
Oxygen administration to immature neonates suppresses VEGF-A expression in the retina, resulting in the catastrophic vessel loss that initiates retinopathy of prematurity. To investigate the mechanisms responsible for survival of blood vessels in the developing retina, we characterized two VEGF-A receptors, VEGF receptor-1 (VEGFR-1, also known as Flt-1) and VEGF receptor-2 (VEGFR-2, also known as Flk-1). Surprisingly, these two VEGF-A receptors differed markedly during normal retinal development in mice. At 5 days postpartum (P5), VEGFR-1 protein was colocalized with retinal vessels, whereas VEGFR-2 was detected only in the neural retina. Real-time RT-PCR identified a 60-fold induction of VEGFR-1 mRNA in retina from P3 (early vascularization) to P26 (fully vascularized), and no significant change in VEGFR-2 mRNA expression. Placental growth factor-1 (PlGF-1), which exclusively binds VEGFR-1, decreased hyperoxia-induced retinal vaso-obliteration from $22.2 \%$ to $5.1 \%$, whereas VEGF-E, which exclusively binds VEGFR-2, had no effect on blood vessel survival. Importantly, under the same conditions, PIGF-1 did not increase vasoproliferation during (a) normal vessel growth, (b) revascularization following hyperoxia-induced ischemia, or (c) the vasoproliferative phase, indicating a selective function supporting blood vessel survival. We conclude that VEGFR-1 is critical in maintaining the vasculature of the neonatal retina, and that activation of VEGFR- 1 by PlGF-1 is a selective strategy for preventing oxygen-induced retinal ischemia without provoking retinal neovascularization.
\end{abstract}

J. Clin. Invest. 112:50-57 (2003). doi:10.1172/JCI200317808.

\section{Introduction}

Retinopathy of prematurity (ROP), a major cause of blindness in children, is a retinal vascular disease associated with oxygen supplementation in the premature infant (1). ROP occurs in two phases (2), as do other retinopathies, and VEGF-A has been shown to be critical to both phases in animal models. In the first phase, byperoxia-induced retinal vessel loss and cessation of vessel growth is accompanied by suppression of VEGF-A expression. This process can be partially prevented by intravitreal injections of VEGF-A in animal models (3, 4). In the second phase, lack of retinal vessels causes bypoxia, which strongly induces VEGF-A expression and stimulates pathological vasoproliferation at the junction of the avascular and vascularized retina $(3,5)$. Thus, VEGF receptors play a critical role in both vasoproliferation and protection from vaso-obliteration.

Received for publication January 9, 2003, and accepted in revised form March 25, 2003.

Address correspondence to: Lois E.H. Smith, Department of Ophthalmology, Children's Hospital Boston, 300 Longwood Avenue, Boston, Massachusetts 02115, USA. Phone: (617) 355-6499; Fax: (617) 734-5731;

E-mail: lois.smith@tch.harvard.edu.

Conflict of interest: The authors have declared that no conflict of interest exists.

Nonstandard abbreviations used: retinopathy of prematurity (ROP); VEGF receptor-1 (VEGFR-1, also known as Flt-1); VEGF receptor-2 (VEGFR-2, also known as Flk-1); placental growth factor (PlGF); postnatal day (P); balanced salt solution (BSS).
If the vasoproliferative and vasoprotective functions of VEGF receptors are separable, it will be possible to devise therapies for newborns that promote retinal capillary survival without inducing vasoproliferation. Such therapies would likely be of considerable importance in preventing ROP. Therefore, we characterized the VEGF-A receptor tyrosine kinases, VEGF receptor-1 (VEGFR-1, also known as Flt-1) and VEGF receptor-2 (VEGFR-2, also known as Flk-1), in neonatal mouse retina and investigated the individual involvement of VEGFR-1 and VEGFR-2 in oxygen-induced retinal vessel degeneration. By using the VEGFR-1-specific ligand human placental growth factor-1 (PlGF-1), which does not bind to neuropilin or VEGFR-2 $(6,7)$, and the VEGFR-2-specific ligand VEGF-E, which does not bind to neuropilin or VEGFR-1 $(8,9)$, we were able to show that specific activation of VEGFR-1 but not VEGFR-2 protects against oxygen-induced vessel loss without stimulating vascular proliferation and neovascularization in vivo. Thus VEGFR-1 is a potential new target for control of vessel degeneration in ROP and perhaps other retinopathies.

\section{Methods}

FITC-dextran perfusion and retinal whole mount. C57BL/6 mice were anesthetized with Avertin (Sigma-Aldrich, St. Louis, Missouri, USA) and sacrificed by intracardiac perfusion with $4 \%$ paraformaldehyde and FITC-dex- 

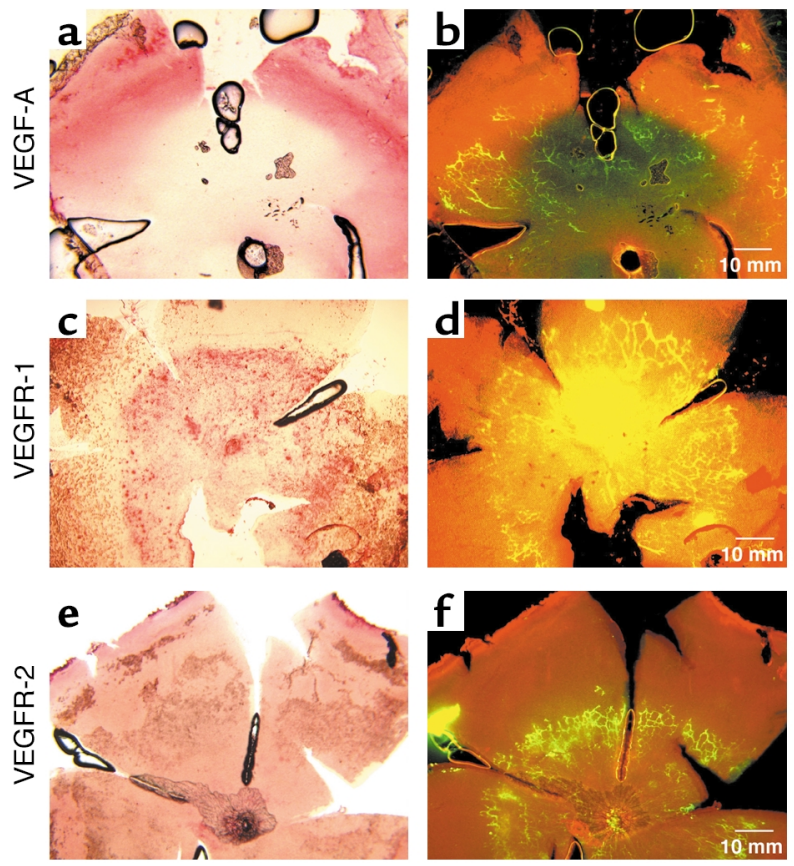

tran (mol wt, $\left.2 \times 10^{6}\right)$ in PBS (10). Eyes were enucleated and fixed in $4 \%$ paraformaldehyde for 2 hours at $4^{\circ} \mathrm{C}$. The retinas were isolated and either directly wholemounted with glycerol-gelatin (Sigma-Aldrich) onto polylysine-coated slides with the photoreceptor side up, or mounted after in situ hybridization or immunohistochemical staining. The retinas were examined with a fluorescence microscope (Olympus Optical Co., Tokyo, Japan). Images were digitized using a 3 CCD color video camera (DX-950P; Sony Corp., Tokyo, Japan) and processed with Northern Eclipse software (Empix Imaging Inc., Toronto, Ontario, Canada).

$\mathrm{O}_{2}$-induced vessel degeneration. To induce vessel loss, postnatal day 7 (P7) C57BL/6 mice with their nursing mothers were exposed to $75 \%$ oxygen for periods ranging from 17 hours to 5 days (11). At P7, $0.5 \mu \mathrm{g}$ human PlGF- 1 in $0.5 \mu$ l balanced salt solution (BSS) (R\&D Systems Inc., Minneapolis, Minnesota, USA) or VEGF-E $(0.5 \mu \mathrm{g} / 0.5 \mu \mathrm{l}$ BSS; Cell Sciences, Norwood, Massachusetts, USA) was intravitreally injected into one eye. Control $(0.5 \mu \mathrm{lBSS})$ was injected into the contralateral eye $(n=6)$. After $\mathrm{O}_{2}$ exposure, eyes were perfused with FITC-dextran and collected, and retinas were wholemounted onto slides as described.

\section{Figure 2}

Real-time RT-PCR quantification of VEGFR-1 and VEGFR-2 mRNA during retinal vascular development. Copy numbers of VEGFR-1 and VEGFR- 2 mRNA $/ 10^{6}$ copies cyclophilin A control mRNA at specific timepoints were measured. (a) VEGFR-1 mRNA expression increases linearly with retinal vascular development; expression is 60-fold higher at P26 than at P3. (b) VEGFR-2 mRNA expression decreases modestly $(<15 \%)$ during retinal vessel development. The ratio of VEGFR-2 mRNA to VEGFR-1 mRNA expression ranges from 200-fold at P3 to twofold at P26.

\section{Figure 1}

P5 retinal whole-mount in situ hybridization of VEGF-A, VEGFR-1, and VEGFR-2 mRNA's. (a) VEGF-A mRNA (pink) is detected anterior to the growing vessel front as outlined by (b) FITC-dextran perfusion of vessels (yellow green). Posterior to the vessel front, VEGF-A expression is suppressed (light yellow). (c) VEGFR-1 mRNA (pink) is detected in the central retina but is not seen at the anterior edge of vessels as outlined by (d) FITC-dextran perfusion of vessels (yellow). (e) VEGFR-2 mRNA (pink) is detected in the entire retina and does not correspond to the vessels ( $f$ ) outlined in yellow.

Whole-mount in situ bybridization. Sense (control) and antisense mRNA probes for VEGF-A, VEGFR-1, and VEGFR-2 were transcribed in vitro using a digoxigenin-UTP labeling kit according to the manufacturer's protocol (Roche Molecular Biochemicals, Indianapolis, Indiana, USA). Retinas were preincubated with $0.2 \mathrm{M} \mathrm{HCl}$ to remove endogenous alkaline phosphatase activity, digested with proteinase K (20 $\mu \mathrm{g} / \mathrm{ml}$ ) in PBS buffer, postfixed in $4 \%$ paraformaldehyde-PBS, and treated with $0.1 \mathrm{M}$ triethanolamine containing $0.25 \%$ acetic anhydride. Retinas were prehybridized in 50\% formamide containing dextran sulfate, ssDNA, and tRNA in phosphate buffer ( $\mathrm{pH}$ 7.5) for 1 hour at $50^{\circ} \mathrm{C}$ and then hybridized with 100 $\mathrm{ng} / \mathrm{ml}$ digoxygenin-labeled RNA probe at $50^{\circ} \mathrm{C}$ overnight. Antibody to digoxygenin (1:1,000; Roche Molecular Biochemicals) was applied for 4 hours at room temperature and color was developed with alkaline phosphatase substrate (Roche Molecular Biochemicals) for 10 minutes at room temperature. Retinas were flat-mounted as described.

Immunohistochemical staining. Eyes were fresh frozen in OCT (Fisher Scientific Co., Pittsburgh, Pennsylvania, USA), cut into $14-\mu \mathrm{m}$ sections, fixed with methanol, rinsed with PBS, and then blocked in PBS with $0.5 \%$ Triton $\mathrm{X}-100$ and $1 \%$ BSA. The sections were stained with primary antibodies against VEGFR-1 or VEGFR-2 (R\&D Systems Inc.), and biotinylated

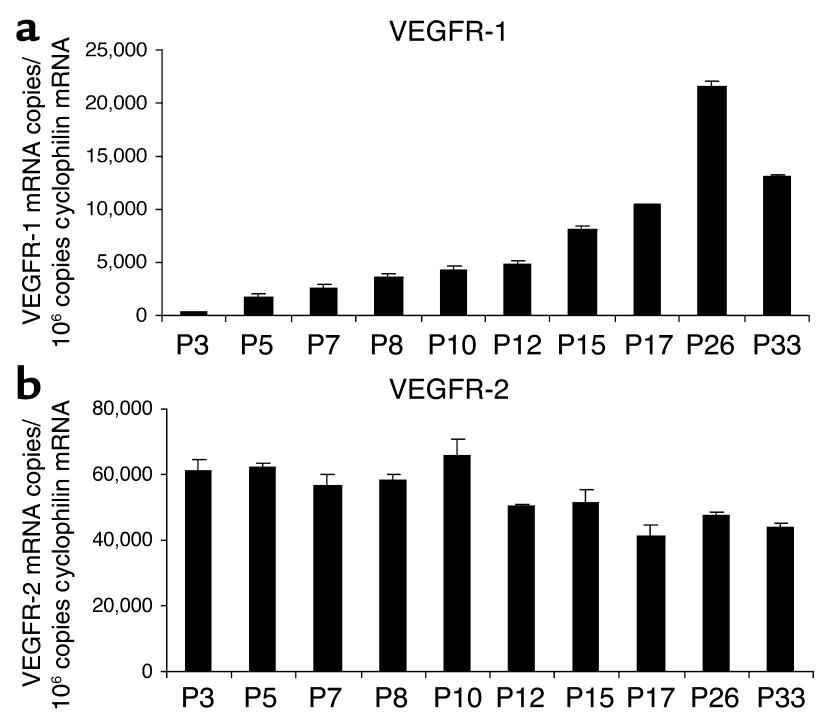


Griffonia simplicifolia, thereafter G. simplicifolia, lectin I (endothelial cell specific) (Vector Laboratories Inc., Burlingame, California, USA) for 2 hours at room temperature. The secondary reagents used were anti-goat-cy3, anti-goat-FITC (Sigma-Aldrich), and avidin-7-amino-4-methylcoumarin-3-acetic acid or avidin-Texas red (Vector Laboratories Inc.). For whole-mount immunohistochemical staining, retinas fixed in $4 \%$ paraformaldehyde for 2 hours were rinsed in PBS, then blocked and stained according to procedures described for cross sections. Images were captured with a 3 CCD color Sony video camera (DX950P) and processed with Northern Eclipse software.

RNA isolation and $c D N A$ preparation. For each selected developmental timepoint (P3, P5, P7, P8, P10, P12, P15, $\mathrm{P} 17, \mathrm{P} 26$, and P33), total RNA was extracted (using an RNeasy kit; QIAGEN Inc., Chatsworth, California, USA) from the retinas of one mouse from each of 12 litters and then pooled to reduce biologic variability. Retinas from each timepoint were lysed in guanidinium isothiocyanate lysis buffer following the manufacturer's instructions and RNA was suspended in diethyl pyrocarbonate-treated $\mathrm{H}_{2} \mathrm{O}$. To generate cDNA, $1 \mu \mathrm{g}$ total RNA was treated with DNase I (Ambion Inc., Austin, Texas, USA) to remove any contaminating genomic DNA. The DNase-treated RNA $(100 \mathrm{ng})$ was then converted into cDNA using murine leukemia virus reverse transcriptase (Invitrogen Corp., San Diego, California, USA). All cDNA samples were aliquoted and stored at $-80^{\circ} \mathrm{C}$.

Quantitative real-time RT-PCR analysis of gene expression. Real-time PCR primers (Genemed Synthesis Inc., South San Francisco, California, USA) targeting murine

\section{Figure 3}

Immunohistochemical localization of VEGFR-1 and VEGFR-2 protein in P2 and P5 retinal whole mounts. On P5, VEGFR-1 protein (red) is detected in a vascular pattern ( $\mathbf{a}$ and $\mathbf{c}$ ) and clearly coincides with endothelial cells (blue) as detected with G. simplicifolia I isolectin (d). (e) A merged image (purple) shows coincidence. ( $b$ and $\mathbf{f}$ ) VEGFR-2-positive signal (red) is found in the neural retina, specifically in the interstices between vessels (blue) (g) in the merged image (h), which shows little or no overlap between vessels and VEGFR-2 staining. On P2, VEGFR-1 protein signal (green) (i) coincides with endothelial cells (red) (j) detected by lectin. In a merged image $(\mathbf{k})$, retinal vessels coincide with the faint VEGFR-1 staining. The retinal vessel front is defined by an arrow. Some hyaloid vasculature $(\mathrm{H})$ from incomplete removal on the vitreal surface of the $\mathrm{P} 2$ retina is also positive for VEGFR-1 signal. (I) VEGFR-2-positive signal (green) is found in the neural retina and does not coincide with endothelial capillaries (red) ( $\mathbf{m})$ in the merged image $(\mathbf{n})$. Arrows indicate the vessel front.
VEGFR-1, VEGFR-2, and cyclophilin A were designed using Primer Express software (Applied Biosystems, Foster City, California, USA). Cyclophilin A expression was unchanged during retinal development and was used as the reference standard (normalizer). Specificity of each primer was determined with NIH's National Center for Biotechnology Information BLAST module. Efficacy of each primer set was assured by testing amplicons for their specific melting-point temperatures using Primer Express software. The ABI Prism 7700 Sequence Detection System and the SYBR Green Master Mix kit ( both from Applied Biosystems) were used for detecting real-time PCR products from reverse-transcribed cDNA samples (0.25-2.5 ng) (12).

To determine absolute copy numbers of murine VEGFR-1 and VEGFR-2 mRNA, we cloned and isolated individual cDNA templates of VEGFR-1, VEGFR-2, and cyclophilin $A$ that cover the sequences bracketed by the real-time PCR primers and determined absolute mRNA numbers as described $(12,13)$. Standard curves for each gene were plotted with quantified cDNA template during each real-time PCR reaction. Each target gene mRNA copy number was then normalized to $10^{6}$ copies of cyclophilin A control.
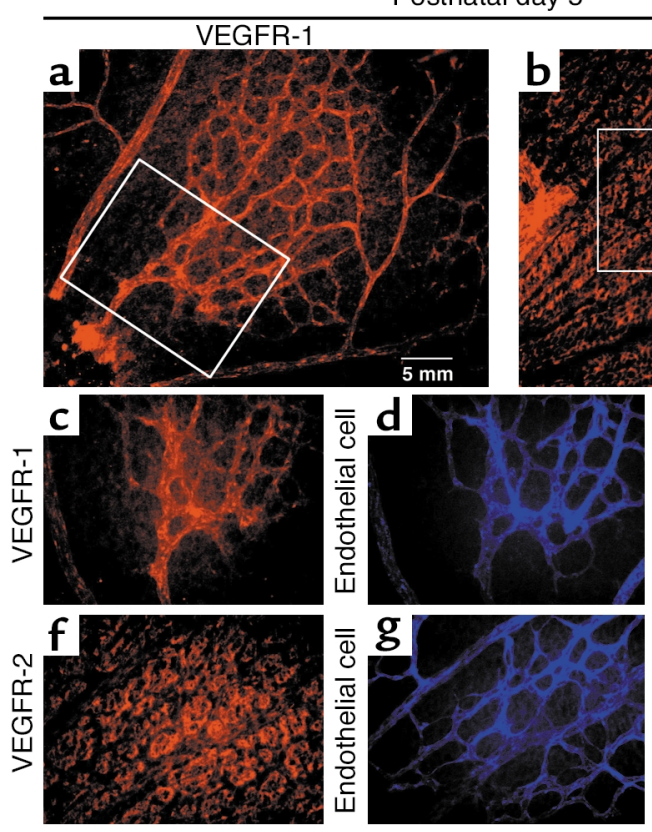

Postnatal day 2
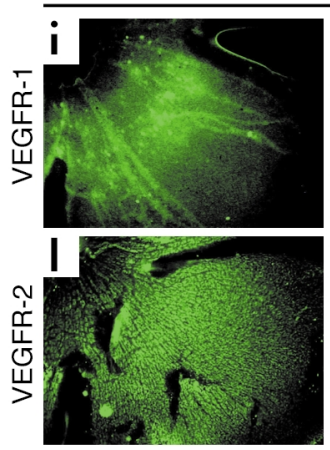
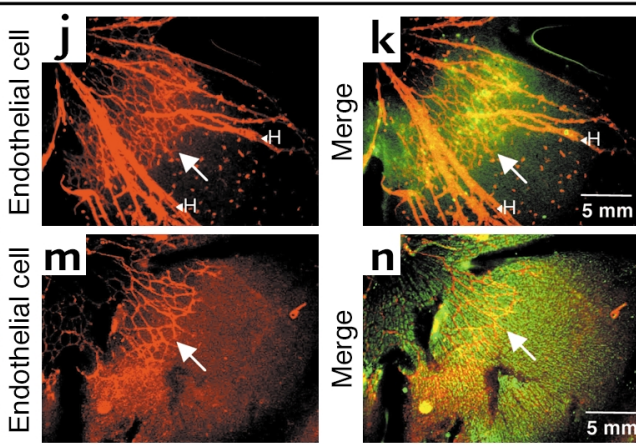
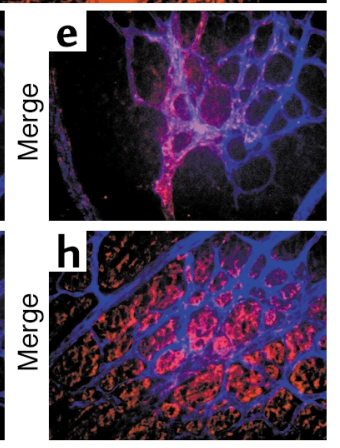

VEGFR-2

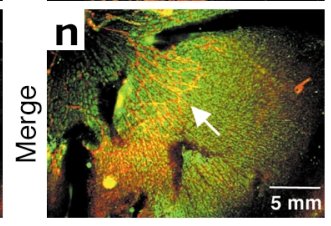



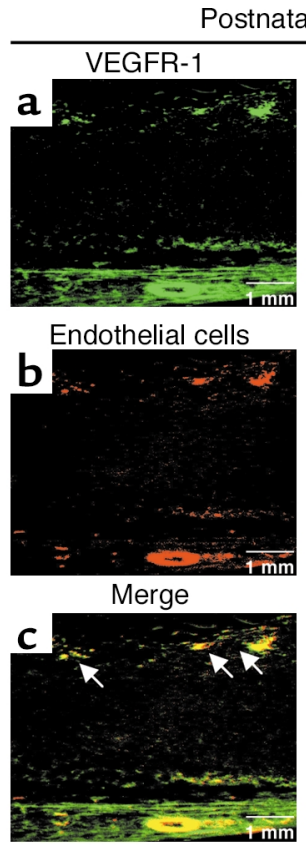

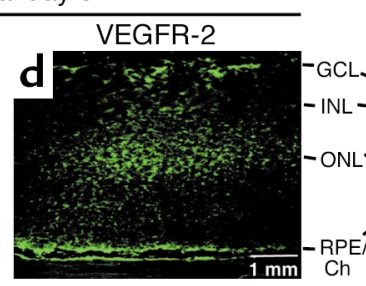

Endothelial cells

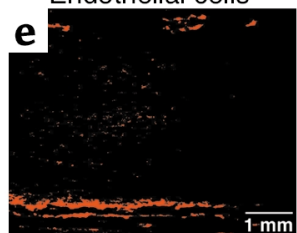

Merge

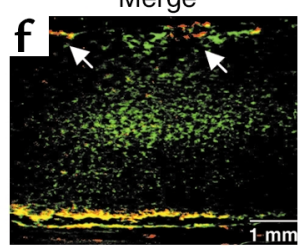

Postnatal day 15
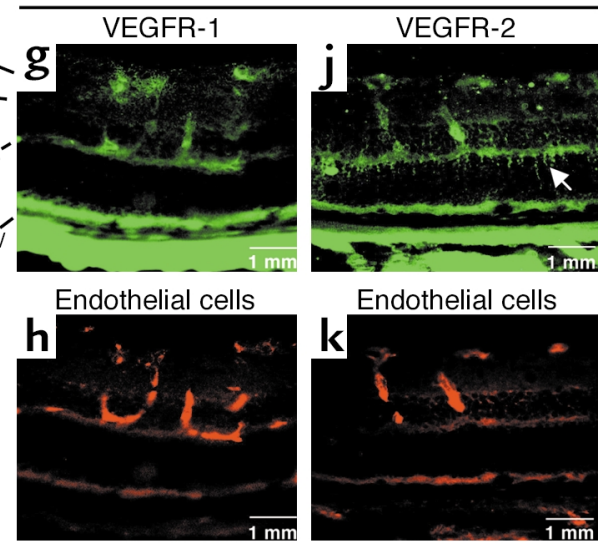

Merge
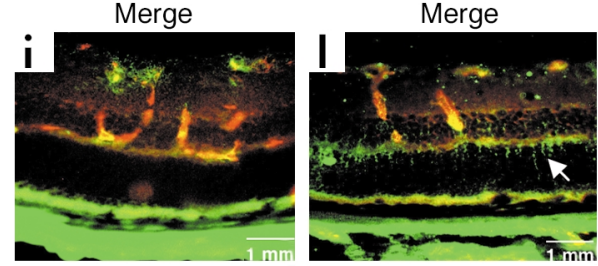

\section{Figure 4}

Immunohistochemical localization of VEGFR-1 and VEGFR-2 protein in P5 and P15 retinal cross sections. (a-f) P5 immunohistochemical localization of (a) VEGFR-1, (d) VEGFR-2, (b and $\mathbf{e})$ G. simplicifolia I isolectin-stained endothelial cells, and (c and $\mathbf{f})$ merged images. (a) VEGFR-1 protein (green) is seen primarily in the ganglion cell layer (GCL) and is seen to overlap with (b) endothelial cells (red) when (c) the images are merged (yellow; indicated by arrows). (d) VEGFR-2-positive signal (green) is found in the GCL, the inner plexiform layer, the inner nuclear layer (INL), and the outer nuclear layer (ONL). (e) Vascular endothelial cells (red) do not overlap with VEGFR-2-positive cells in the merged image (f) and are not coincident with vessels (arrows). (g-I) P15 immunohistochemical localization of (g) VEGFR-1 and (j) VEGFR-2 staining and (h and $\mathbf{k}$ ) isolectin-stained endothelial cells. ( $\mathbf{i}$ and $\mathbf{I}$ ) Merged images. VEGFR-1 protein (green in $\mathbf{g}$ ) completely overlaps with endothelial cells (red in $\mathbf{h}$ ) when the images are merged (yellow in $\mathbf{i}$ ). Some VEGFR-2 signal (green in $\mathbf{j}$ ) overlaps with endothelial cells $(\mathbf{k})$ when the images are merged (yellow in $\mathbf{I}$ ), whereas other VEGFR-2-positive cells (indicated by arrow in $\mathbf{d}$ and $\mathbf{f}$ ) span the retina and are morphologically consistent with Muller cell structure. RPE/Ch, retinal pigment epithelium/choroid.

The sequences of the PCR primer pairs $\left(5^{\prime}\right.$ to $\left.3^{\prime}\right)$ that were used for each gene were: VEGFR-1, $5^{\prime}$-GAGGAGGATGAGGGTGTCTATAGGT- $3^{\prime}$ (forward) and $5^{\prime}$-GTGATCAGCTCCAGGTTTGACTT-3' (reverse); VEGFR-2, $5^{\prime}$ GCCCTGCCTGTGGTCTCACTAC- $3^{\prime}$ (forward) and $5^{\prime}$ CAAAGCATTGCCCATTCGAT- $3^{\prime}$ (reverse); cyclophilin A, 5'-CAGACGCCACTGTCGCTTT-3' (forward) and 5'-TGTCTTTGGAACTTTGTCTGCAA-3' (reverse).

Analysis of retinal vessel growth and retinal revascularization

Early retinal vessel development. At $\mathrm{P} 3$, PlGF-1 $(0.5 \mu \mathrm{g} / 0.5$ $\mu \mathrm{l}$ BSS) was injected into the vitreous of one eye and control $(0.5 \mu \mathrm{lBSS})$ was injected into the contralateral eye $(n=6)$. Forty-eight hours later (at P5) retinas were harvested after FITC-dextran perfusion (10) and wholemounted. Images were captured with a color video camera and vessel growth was measured using Northern Eclipse software and expressed as a percentage of total retinal area (14). To show that the system could detect an increase in vascular growth with cytokine stimulation, we examined a positive control (12) consisting of cells expressing high levels of VEGF-A injected into one eye and saline into the contralateral eye of mice as follows: (a) injection at P7 followed by flat- mounting of retina at $\mathrm{P} 12(n=6),(\mathrm{b})$ injection at $\mathrm{P} 12$ followed by flat-mounting at P15 $(n=6)$, and (c) injection at P15 followed by retinal flat-mounting at P17 $(n=6)$. VEGF-A significantly increased vessel density with exposure from P7-P12, showing that the method could detect a positive change (data not shown).

Retinal revascularization after oxygen-induced vessel loss. P7 mice were exposed to $75 \% \mathrm{O}_{2}$ for 5 days to induce vessel loss and returned to room air at P12 (11). After 24 hours (P13), mice were injected intravitreally with PlGF- $1(0.5 \mu \mathrm{g} / 0.5 \mu \mathrm{l} \mathrm{BSS})$ in one eye and with $0.5 \mu \mathrm{l}$ BSS in the contralateral eye $(n=6)$. Retinas were isolated at P15 after FITC-dextran perfusion and wholemounted. Vascularized area was measured as above to determine the effect of PlGF-1 on revascularization. Positive control retinal flat mounts showed a significant increase in vessel density at P15 after VEGF-A treatment at P12 (data not shown).

Retinal neovascularization after oxygen-induced vessel loss. After 5 days of oxygen exposure, from P7 to P12 (11), P13 mice were injected intravitreally with PlGF-1 (0.5 $\mu \mathrm{g} / 0.5 \mu \mathrm{l} \mathrm{BSS})$ in one eye and with control (0.5 $\mu \mathrm{l} \mathrm{BSS})$ in the contralateral eye $(n=8)$. Retinas were harvested at P17 and the mean numbers of neovascular nuclei extending into the vitreous were counted in retinal 
cross sections as previously described $(5,11,14)$. Positive-control retinal flat mounts showed a significant increase in vessel growth at P17 with VEGF-A treatment at P15 (data not shown).

\section{Results}

VEGFR-1, VEGFR-2, and VEGF-A mRNA expression during retinal vessel development. Early in retinal vascular development, at P5, whole-mount in situ hybridization identified VEGF-A mRNA expression in areas of physiological hypoxia anterior to the growing vessel front. Suppression of VEGF-A expression coincided with retinal vessel formation (Figure 1, a and b). In contrast, VEGFR-1 mRNA expression coincided with more maturing retinal vessels (Figure 1, $\mathrm{c}$ and $\mathrm{d}$ ), whereas VEGFR-2 mRNA expression was uniform over the entire retina (Figure 1, e and $\mathrm{f}$ ). Hybridization with control sense mRNAs for VEGF-A, VEGFR-2, and VEGFR-1 showed no significant background (data not shown). At P7, a time when superficial retinal vessels have extended further into the periphery, VEGF-A mRNA was observed anterior to vessels in the same pattern as that observed at P5 (data not shown). Similarly, the pattern seen at P5 with VEGFR-1 and VEGFR-2 was also seen at P7 (data not shown).

VEGFR-1 and VEGFR-2 mRNA expression from whole retina during vessel development was examined by quantitative real-time RT-PCR. VEGFR-1 mRNA expression increased linearly during the course of retinal vessel development (Figure 2a). At P3, when retinas are still largely avascular, 350 copies of VEGFR- $1 \mathrm{mRNA} / 10^{6}$ copies of cyclophilin A (internal control) were detected. By P26, when retinal vessels are nearly fully developed, VEGFR-1 mRNA expression had increased 60 -fold $(22,000$ copies VEGFR-1 mRNA $/ 10^{6}$ copies of cyclophilin A mRNA). Expression declined at P33 when retinal

\section{Figure 5}

PIGF-1, but not VEGF-E, prevents hyperoxia-induced retinal vessel loss, thus implicating VEGFR-1 in survival. PIGF-1: P8 FITCdextran-perfused retinal flat-mount retina from a representative control mouse treated with room air (normoxia) (a) or a mouse given hyperoxic treatment $\left(75 \% \mathrm{O}_{2}\right.$ for 17 hours at P7-P8) after intravitreal injection on P7 of (b) control BSS in one eye and (c) the VEGFR-1-specific ligand PIGF-1 in the contralateral eye. Vessels delineated with FITC show that PIGF-1 confers significant protection from oxygen-induced vessel loss compared with BBS control. (d) Analysis of nonvascularized area shows a greater than fourfold difference between eyes treated with PIGF-1 $(22.2 \% \pm 3.4 \%$ vascularized area) and eyes treated with BSS $(5.1 \% \pm 1.2 \%)(n=6, P<0.001)$. VEGF-E: FITC-dextran-perfused retinal flat mount of $\mathrm{P} 8$ control retina from representative room air-treated mouse (e) or oxygen-exposed mouse after intravitreal injections at P7 of $(\mathbf{f})$ control BSS in one eye and $(\mathbf{g})$ VEGFR-2-specific ligand VEGF-E in the contralateral eye. (h) Analysis of nonvascularized area shows no significant difference between VEGF-E- and BSS-treated eyes $(n=6, P=0.87)$. Results are representative of two independent experiments. vessels were fully developed, falling to 13,000 copies VEGFR-1 mRNA/ $10^{6}$ copies of cyclophilin A mRNA. This result provides further evidence of a relationship between VEGFR-1 expression and retinal vessel formation. Quantification of VEGFR-2 mRNA revealed sharp contrasts with VEGFR-1 mRNA expression. Early in retinal vascular development (P3), VEGFR-2 mRNA expression was about 170-fold higher than VEGFR-1 (60,000 VEGFR-2 versus 350 VEGFR- 1 mRNA copies $/ 10^{6}$ copies of cyclophilin A mRNA). Moreover, this ratio declined with retinal vascular development and reached a ratio of about 2:1 when retinas were fully vascularized, at P26 (45,000 versus 22,000 VEGFR-2 mRNA copies/10 copies of cyclophilin A mRNA) (Figure 2b).

Immunohistochemical localization of VEGFR-1 and VEGFR-2 proteins in developing retina. In retinal whole mounts of P2 and P5 mice, VEGFR-1, VEGFR-2, and endothelial cells were visualized with immunohistochemical staining with VEGFR-1 antibody, VEGFR-2 antibody, and G. simplicifolia I isolectin, respectively. VEGFR-1 antibody staining coincided with retinal vessels at P5 (Figure 3a), whereas VEGFR-2 antibody primarily stained nonvascular cells of the neural retina (Figure 3b). In merged images, the VEGFR-1-positive cells completely overlapped with isolectin-positive retinal vessels (Figure 3, c-e). In contrast, VEGFR-2-positive cells appeared in the interstices between isolectinpositive retinal vessels (Figure 3, f-h), indicating that
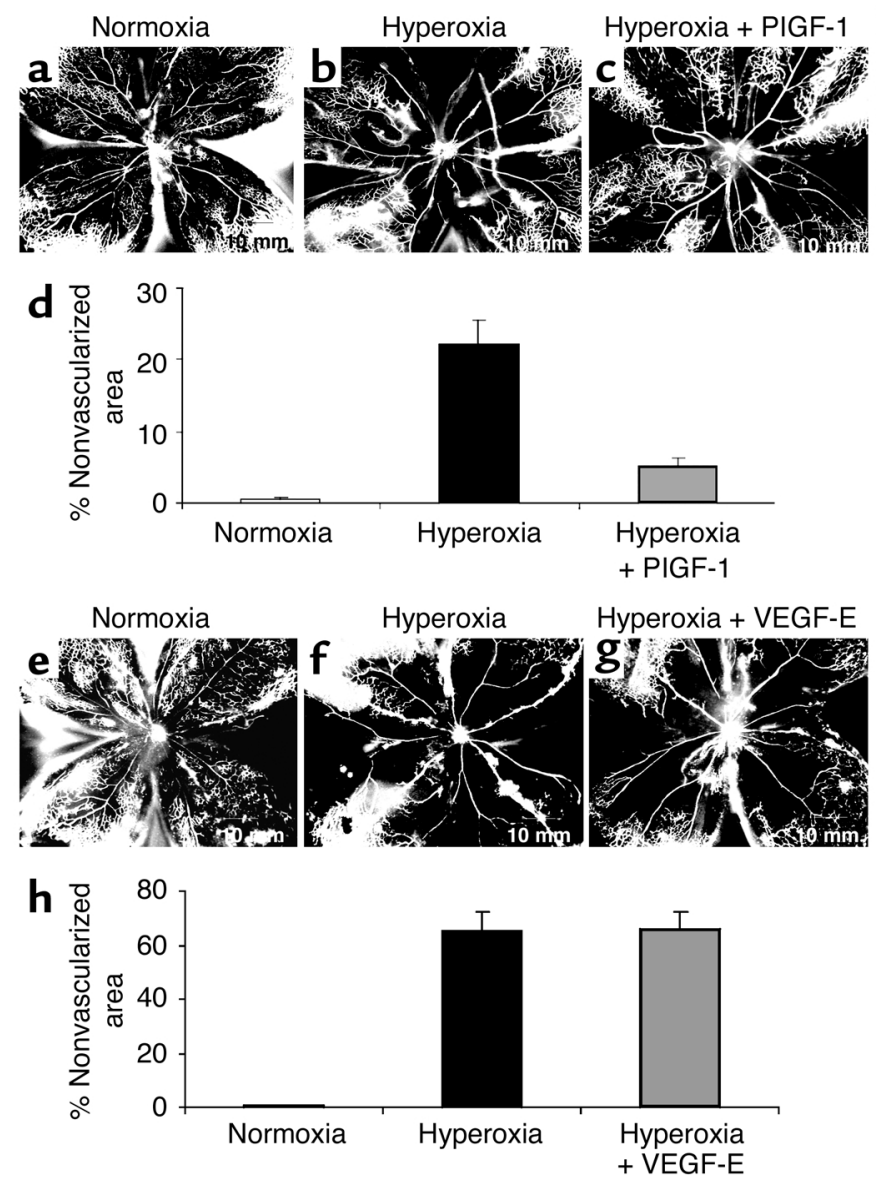


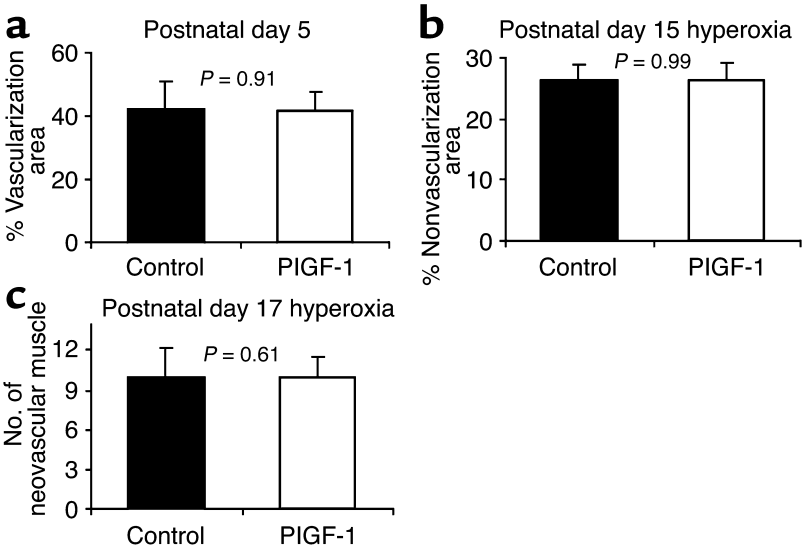

Figure 6

Activation of VEGFR-1 by PIGF-1 does not increase normal retinal vessel growth or revascularization. (a) Intravitreal injections at P3 of control (BSS) in one eye and PIGF-1 in the contralateral eye $(n=6)$. Retinal vessel growth area was measured in whole mounts at P5. PIGF-1-injected eyes had a mean of $41.67 \% \pm 5.63 \%$ of the retina vascularized. Similarly, $42.18 \% \pm 8.60 \%$ of the BSS-injected contralateral control eyes were vascularized $(P=0.91)$. Results are representative of two independent experiments. (b) Vessel revascularization was measured in P15 mice after induction of vessel loss by oxygen (P7-P12) followed by intravitreal injections of BSS at P13 in one eye and PIGF-1 in the contralateral eye. PIGF-1-injected eyes were $26.32 \% \pm 2.62 \%$ vascularized; similarly, BSS-treated contralateral control eyes were $26.29 \% \pm 2.86 \%$ vascularized $(n=6, P=0.99)$. Results are representative of two independent experiments. (c) In eyes with oxygen-induced retinopathy, the mean number of vascular nuclei extending into the vitreous at $\mathrm{P} 17$ in ten retinal cross sections per eye ( $n=8$ eyes) was counted after intravitreal injections of BSS at $\mathrm{P} 13$ (after 5 days of $75 \% \mathrm{O}_{2}$ treatment, from $\mathrm{P} 7$ to $\mathrm{P} 12$ ) in one eye and PIGF-1 in the contralateral eye. BSS- and PIGF-1-injected eyes showed means of 9.98 and 9.96 vascular nuclei $(P=0.61)$, respectively, indicating no stimulation of proliferation by PIGF-1.

VEGFR-2 expression is primarily associated with the neural retina. This vascular endothelial-specific expression of VEGFR-1 (Figure 3g) and neural retinal-specific expression of VEGFR-2 (Figure 31) can be detected as early as P2, at which time endothelial cells identified with isolectin completely coincided with VEGFR-1 staining (Figure $3, \mathrm{j}$ and $\mathrm{k}$ ). As early as P2, VEGFR-2 was found in the neural retina alone, in the interstices between retinal vessels (Figure 3, $\mathrm{m}$ and $\mathrm{n}$ ).

To confirm these findings, we examined P5 VEGFR-1 and VEGFR-2 protein localization in retinal cross section. VEGFR-1 antibody prominently stained cells in the ganglion cell layer (Figure 4a). Staining coincided with vascular endothelial cells identified with endothelial cell-specific isolectin. (Figure 4, b and c). VEGFR-2 antibody also stained cells prominently in the ganglion cell layer and the inner and outer nuclear cell layers of P5 retinal cross sections (Figure 4, d-f). However, VEGFR-2 antibody staining did not colocalize with vascular endothelial cells but instead localized to neural retina, thus verifying our findings in whole mounts. This neural cell-specific expression of VEGFR-2 was limited to perinatal mice. By P12, when blood vessels were more fully developed into three layers, anti-VEGFR-2 staining began to coincide with some (but not the majority of) vascular endothelial cell staining (as well as with VEGFR-1). VEGFR-2 was still found in neural retina (data not shown). By P15, vascular staining with VEGFR-2 became more evident (Figure 4, $j-1$ ), as VEGFR-1 continued to be specifically expressed on endothelial cells (Figure 4, g-i). VEGFR-2 can be seen on cells spanning the thickness of the retina, consistent with Muller cell morphology (Figure 4, $\mathrm{j}$ and $\mathrm{l}$; indicated by arrow). Overall, these results indicate that in neonatal retina, VEGFR-1 expression is specific to retinal blood vessels, whereas VEGFR-2 is predominantly expressed in neural cells and is developmentally regulated in blood vessels. Only with maturation is VEGFR-2 (along with VEGFR-1) also expressed in blood vessels.

VEGFR-1-specific agonist PlGF-1 protects against oxygeninduced vessel loss; VEGFR-2-specific agonist VEGF-E is not protective. PlGF- 2 is the only PlGF isoform produced in the mouse. It is a ligand for VEGFR-1 but also a ligand for neuropilin (15), a VEGF receptor associated with angiogenesis (16). In order to define specific functions of VEGFR-1 and VEGFR-2 in hyperoxia-induced retinal vessel loss, we administered the VEGFR-1-specific ligand PIGF-1 $(6,7)$ and the VEGFR-2-specific ligand VEGF-E $(8,9)$ by intravitreal injection. PlGF-1 does not bind to neuropilin, unlike PlGF-2. VEGF-E binds only VEGFR-2 and does not bind neuropilin. Thus with the use of these specific ligands we could eliminate confounding effects of neuropilin binding.

Analysis of vascularized/nonvascularized area indicated a reduction of approximately $77 \%$ in hyperoxiainduced vessel loss associated with PlGF-1 intravitreal injections in comparison with controls (Figure 5, a and d). Specifically, eyes given hyperoxic treatment for 17 hours had nonvascularized area of $22.2 \% \pm 3.4 \%$ (Figure 5, b and d), whereas contralateral PlGF-1injected eyes showed only $5.1 \% \pm 1.2 \%$ nonvascularized area $(P<0.0018)$ (Figure $5, \mathrm{c}$ and $\mathrm{d})$. In contrast, VEGF-E provided no detectable protective effect against $\mathrm{O}_{2}$-induced vessel loss compared with control BSS-injected contralateral eyes (Figure 5, e-h). Thus, these experiments demonstrate that PlGF-1, which specifically binds VEGFR-1 and no other VEGFR, protects postnatal mouse retina from hyperoxia-induced vaso-obliteration, whereas the specific activation of VEGFR-2 by VEGF-E is not protective.

PlGF-1 does not stimulate murine retinal vessel growth. Because PlGF-1 at low concentrations protected against hyperoxia-induced retinal vessel loss, we explored whether PlGF-1 at similar concentrations also stimulated retinal vessel growth in vivo under conditions of normal vessel development and during regrowth of vessels after $\mathrm{O}_{2}$-induced vessel loss at P15. Intravitreal administration of PlGF-1 $(0.5 \mu \mathrm{g} / 0.5 \mu \mathrm{l}$ BSS) at P3 resulted in no significant stimulation of normal vessel growth compared with control eyes when 
examined 2 days later at P5 $(n=6, P=0.91$; Figure $6 a)$. In addition, administration of PlGF-1 at P13, after 5 days of $75 \% \mathrm{O}_{2}$ exposure (from P7 to P12), did not stimulate subsequent regrowth of vessels as measured at P15 ( $P=0.99$; Figure 6b). Similarly, intravitreal injections of PlGF-1 at P13 also did not affect retinal neovascularization measured at P17 (Figure 6c). These findings indicate that PlGF-1, at concentrations that prevent vessel loss, does not stimulate retinal vascular proliferation and neovascularization in vivo.

\section{Discussion}

Although VEGF-A is known to promote survival of retinal vessels under hyperoxia, the role of specific VEGF-A receptors in this process has not been described. Using receptor-specific ligands, this study has distinguished the contributions of VEGF-A receptors VEGFR-1 and VEGFR-2 to the survival of retinal blood vessels in neonatal mice. Specifically, we find that PlGF-1, a VEGFR-1-specific agonist, is critical to vessel survival and does not promote vasoproliferation. These findings suggest the important possibility of treating premature infants with VEGFR-1-specific ligands in order to prevent retinal ischemia without provoking vasoproliferation.

Our study indicates that concentrations of PlGF-1 sufficient to prevent oxygen-induced vessel loss did not increase retinal vessel proliferation during normal retinal vascular development. Nor did such concentrations of PlGF-1 increase regrowth of vessels after oxygeninduced loss or increase hypoxia-induced vasoproliferation. Thus, our findings identify selective functions for PlGF-1 and VEGFR-1 in supporting retinal blood vessel survival in neonates.

VEGFR-1 tyrosine kinase activation is not necessary for normal vascular development, since deletion of the tyrosine kinase domain of VEGFR-1 allows normal embryonic angiogenesis (17). Deletion of the entire VEGFR-1 gene is embryonically lethal due to abnormal vascular development. These abnormalities may be attributable to loss of soluble VEGFR-1, which is a natural competitor for VEGF binding to VEGFR-2 (18). Although at low concentrations we found no increase in vasoproliferation with PlGF-1, some studies suggest that, in other settings, VEGFR-1 may play a role in pathological vasoproliferation (19-21). Our studies suggest that, in some cases, what appears to be VEGFR-1induced increased proliferation may be due to increased vascular survival. In order to specifically define the role of VEGFR-1, we chose to target VEGFR-1 with PlGF-1, which binds VEGFR-1 and does not bind neuropilin, unlike PlGF-2, which binds both receptors. Neuropilin is critically involved in vascular development $(15,16)$, and studies with PlGF-2 are more difficult to interpret because of neuropilin binding.

Consistent with the specific importance of VEGFR-1 for blood vessel survival in neonatal retina, we found that VEGFR-1 is localized to blood vessels but that VEGFR-2 is localized primarily to the neural retina. Our observation that VEGFR-2 is expressed primarily outside the vasculature in the neonatal retina is in accord with a growing body of evidence showing the importance of VEGF receptors in neural cells (14, 22-26). Interestingly, this selective localization of VEGFR-2 expression to the neural retina does not persist. By P12, VEGFR-2 is also seen on retinal vessels. This change of VEGFR-2 expression with retinal development suggests coordinated regulation of VEGF activity toward neural cells and vascular endothelial cells.

In summary, experiments described here identify a prominent association between VEGFR-1 and neonatal blood vessels in retina and establish that specific stimulation of VEGFR-1 with low doses of PlGF-1 protects retinal vessels from oxygen-induced degeneration without promoting vascular growth. In contrast to VEGFR-1, VEGFR-2 is predominantly expressed in neural retina, and specific stimulation of VEGFR-2 with VEGF-E does not prevent oxygen-induced vasoobliteration. Thus, these studies define important distinctions between VEGFR-1 and VEGFR-2 in neonatal retina, and they identify specific stimulation of VEGFR-1 as an attractive strategy for preventing the early degenerative stage of ROP.

\section{Acknowledgments}

We are grateful for support from the V. Kann Rasmussen Foundation and from the NIH (EYO 8670 to L.E.H. Smith).

1. Gibson, D.L., Sheps, S.B., Uh, S.H., Schechter, M.T., and McCormick, A.Q. 1990. Retinopathy of prematurity-induced blindness: birth weightspecific survival and the new epidemic. Pediatrics. 86:405-412.

2. Bossi, E., and Koerner, F. 1995. Retinopathy of prematurity. Intensive Care Med. 21:241-246.

3. Alon, T., et al. 1995. Vascular endothelial growth factor acts as a survival factor for newly formed retinal vessels and has implications for retinopathy of prematurity. Nat. Med. 1:1024-1028.

4. Pierce, E.A., Foley, E.D., and Smith, L.E. 1996. Regulation of vascular endothelial growth factor by oxygen in a model of retinopathy of prematurity [erratum 1997, 115:427]. Arch. Ophthalmol. 114:1219-1228.

5. Aiello, L.P., et al. 1995. Suppression of retinal neovascularization in vivo by inhibition of vascular endothelial growth factor (VEGF) using soluble VEGF-receptor chimeric proteins. Proc. Natl. Acad. Sci. U. S. A. 92:10457-10461.

6. Park, J.E., Chen, H.H., Winer, J., Houck, K.A., and Ferrara, N. 1994. Placenta growth factor. Potentiation of vascular endothelial growth factor bioactivity, in vitro and in vivo, and high affinity binding to Flt- 1 but not to Flk-1/KDR. J. Biol. Chem. 269:25646-25654.

7. Persico, M.G., Vincenti, V., and DiPalma, T. 1999. Structure, expression and receptor-binding properties of placenta growth factor (PIGF). Curr. Top. Microbiol. Immunol. 237:31-40.

8. Meyer, M., et al. 1999. A novel vascular endothelial growth factor encoded by Orf virus, VEGF- E, mediates angiogenesis via signalling through VEGFR-2 (KDR) but not VEGFR-1 (Flt-1) receptor tyrosine kinases. EMBO J. 18:363-374.

9. Ogawa, S., et al. 1998. A novel type of vascular endothelial growth factor, VEGF-E (NZ-7 VEGF), preferentially utilizes KDR/Flk-1 receptor and carries a potent mitotic activity without heparin-binding domain. J. Biol. Chem. 273:31273-31282.

10. D’Amato, R., Wesolowski, E., and Smith, L.E. 1993. Microscopic visualization of the retina by angiography with high-molecular-weight fluorescein-labeled dextrans in the mouse. Microvasc. Res. 46:135-142.

11. Smith, L.E., et al. 1994. Oxygen-induced retinopathy in the mouse. Invest. Ophthalmol. Vis. Sci. 35:101-111.

12. Shih, S.C., et al. 2002. Molecular profiling of angiogenesis markers. Am. J. Pathol. 161:35-41.

13. Calvo, A., et al. 2002. Inhibition of the mammary carcinoma angiogenic switch in C3(1)/SV40 transgenic mice by a mutated form of human endostatin. Int. J. Cancer. 101:224-234. 
14. Robinson, G.S., et al. 2001. Nonvascular role for VEGF: VEGFR-1, 2 activity is critical for neural retinal development. FASEB J. 15:1215-1217.

15. Migdal, M., et al. 1998. Neuropilin-1 is a placenta growth factor-2 receptor. J. Biol. Chem. 273:22272-22278.

16. Takashima, S., et al. 2002. Targeting of both mouse neuropilin-1 and neuropilin-2 genes severely impairs developmental yolk sac and embryonic angiogenesis. Proc. Natl. Acad. Sci. U. S. A. 99:3657-3662.

17. Hiratsuka, S., Minowa, O., Kuno, J., Noda, T., and Shibuya, M. 1998. Flt-1 lacking the tyrosine kinase domain is sufficient for normal development and angiogenesis in mice. Proc. Natl. Acad. Sci. U. S. A. 95:9349-9354.

18. Kendall, R.L., and Thomas, K.A. 1993. Inhibition of vascular endothelial cell growth factor activity by an endogenously encoded soluble receptor. Proc. Natl. Acad. Sci. U. S. A. 90:10705-10709.

19. Carmeliet, P., et al. 2001. Synergism between vascular endothelial growth factor and placental growth factor contributes to angiogenesis and plasma extravasation in pathological conditions. Nat. Med. 7:575-583.

20. Adini, A., Kornaga, T., Firoozbakht, F., and Benjamin, L.E. 2002. Placental growth factor is a survival factor for tumor endothelial cells and macrophages. Cancer Res. 62:2749-2752.

21. Hiratsuka, S., et al. 2001. Involvement of Flt-1 tyrosine kinase (vascular endothelial growth factor receptor-1) in pathological angiogenesis. Cancer Res. 61:1207-1213.

22. Sondell, M., and Kanje, M. 2001. Postnatal expression of VEGF and its receptor flk-1 in peripheral ganglia. Neuroreport. 12:105-108.

23. Witmer, A.N., et al. 2002. Altered expression patterns of VEGF receptors in human diabetic retina and in experimental VEGF-induced retinopathy in monkey. Invest. Ophthalmol. Vis. Sci. 43:849-857.

24. Yang, K., and Cepko, C.L. 1996. Flk-1, a receptor for vascular endothelial growth factor (VEGF), is expressed by retinal progenitor cells. J. Neurosci. 16:6089-6099.

25. Ogata, N., et al. 1998. Expression of vascular endothelial growth factor and its receptor, KDR, following retinal ischemia-reperfusion injury in the rat. Curr. Eye Res. 17:1087-1096.

26. Suzuma, K., Takagi, H., Otani, A., Suzuma, I., and Honda, Y. 1998. Increased expression of KDR/Flk-1 (VEGFR-2) in murine model of ischemia- induced retinal neovascularization. Microvasc. Res. 56:183-191. 